\title{
Catalytic enantioselective Diels-Alder reaction in ionic liquid via a recyclable chiral In(III) complex
}

Fan Fu, Yong-Chua Teo and Teck-Peng Loh * 


\title{
Catalytic enantioselective Diels-Alder reaction in ionic liquid via a recyclable chiral indium(III) complex
}

\author{
Fan Fu, Yong-Chua Teo and Teck-Peng Loh ${ }^{\mathrm{a} *}$ \\ $a^{a^{*}}$ School of Physical and Mathematical Sciences, Division of Chemistry and Biological \\ Chemistry, Nanyang Technological University, 1 Nanyang Walk, Blk 5, Level 3, \\ Singapore 637616
}

\section{Supporting Information}

Table of Contents

Page

General Methods

General Procedure for Enantioselective Diels-Alder Reaction in

Procedure for Enantioselective Diels-Alder Reaction in

Ionic Liquid (recyclability)

Characterization of Diels-Alder adduct 


\section{General Methods}

Experiments involving moisture and/or air sensitive components were performed in oven-dried glassware under a positive pressure of nitrogen using freshly distilled solvents. Commercial grade solvents and reagents were used without further purification with the following exceptions: Dichloromethane was distilled from calcium hydride. Tetrahydrofuran was distilled from sodium. Hexane, ethyl acetate were fractionally distilled. Azeotropic drying of starting materials or reagents was performed by the addition of the stated amount of anhydrous tetrahydrofuran, ensued by azeotropic removal of tetrahydrofuran with traces of moisture in vacuo followed by subsequent purging with nitrogen. 2-Methacrolein was freshly distilled prior to usage. 1,3-Cyclopentadiene was cracked at $170{ }^{\circ} \mathrm{C}$ and re-distilled. 2Bromoacrolein, ${ }^{1}$ 7-methoxy-4-vinyl-1,2-dihydro-napthalene ${ }^{2}$ was prepared according to literature procedures. ${ }^{3}$

Analytical thin layer chromatography (TLC) was performed using Merck 60 F254 precoated silica gel plate $(0.2 \mathrm{~mm}$ thickness $)$. Subsequent to elution, plates were visualized using UV radiation (254 nm) on Spectroline Model ENF-24061/F $254 \mathrm{~nm}$. Further visualization was possible by staining with basic solution of potassium permanganate or acidic solution of ceric molybdate, followed by heating on a hot plate.

Flash chromatography was performed using Merck silica gel 60 with freshly distilled solvents. Columns were typically packed as slurry and equilibrated with the appropriate solvent system prior to use.

1(a) Corey, E. J.; Snider, B. B. J. Am. Chem. Soc. 1972, 94, 2549. (b) Corey, E. J.; Loh, T.-P. J. Am. Chem. Soc. 1991, 113, 8966.

${ }^{2}$ Woski, S.A.; Koreeda, M. J. Org. Chem. 1992, 57, 5736.

${ }^{3}$ Louis, D. Q.; Alan, N. H.; Franklin, L.; Annette, L. G. Tetrahedron 1983, 39, 401. 
Infrared spectra were recorded on a Bio-Rad FTS 165 FTIR spectrometer. Liquid samples were examined as film between $\mathrm{NaCl}$ salt plates.

Proton nuclear magnetic resonance spectra $\left({ }^{1} \mathrm{H}\right.$ NMR) were recorded on a Bruker Avance DPX 300 and Bruker AMX 500 spectrophotometer $\left(\mathrm{CDCl}_{3}\right.$ as solvent). Chemical shifts for ${ }^{1} \mathrm{H}$ NMR spectra are reported as $\delta$ in units of parts per million (ppm) downfield from $\mathrm{SiMe}_{4}(\delta 0.0)$ and relative to the signal of chloroform- $d(\delta 7.2600$, singlet). Multiplicities were given as: s (singlet); bs (broad singlet); d (doublet); bd (broad doublet); t (triplet); q (quartet); dd (doublets of doublet); or m (multiplets). The number of protons (n) for a given resonance is indicated by $\mathrm{nH}$. Coupling constants are reported as a $J$ value in Hz. Carbon nuclear magnetic resonance spectra $\left({ }^{13} \mathrm{C} \mathrm{NMR}\right)$ are reported as $\delta$ in units of parts per million (ppm) downfield from $\mathrm{SiMe}_{4}(\delta 0.0)$ and relative to the signal of chloroform- $d(\delta 77.03$, triplet). The proportion of diastereomers and geometric isomers was determined from the integration of ${ }^{1} \mathrm{H} \mathrm{NMR}$ and ${ }^{13} \mathrm{C}$ NMR spectra.

Mass spectral analyses were carried out on a VG 7035 micromass mass spectrophotometer at a source temperature of $200{ }^{\circ} \mathrm{C}$ and at an ion current of $70 \mathrm{eV}$. Mass spectral data were reported in units of mass to charge $(\mathrm{m} / \mathrm{z})$ and $\%$ intensity. 


\section{General Procedure for Enantioselective Diels-Alder Reaction in ionic liquid}

Representative procedure for enantioselective Diels-Alder reaction : Preparation of (1R $\underline{2 R, 4 R) \text {-2-bromo-bicyclo[2.2.1] hept-5-ene-2-carbaldehyde }}$

To an oven dried $10 \mathrm{~mL}$ round-bottom flask equipped with a magnetic stirring bar was added $\mathrm{InCl}_{3}$ (22 mg, $0.1 \mathrm{mmol}, 0.2$ equiv.). The solid was azeotropically dried with anhydrous tetrahydrofuran twice $(2 \mathrm{~mL} \times 2)$ prior to the addition of $1.5 \mathrm{~mL}$ of dichloromethane. $(S)$ BINOL (31 mg, $0.11 \mathrm{mmol}, 0.22$ equiv.) and $4 \AA$ molecular sieve (15 mg) were added and the mixture was stirred under nitrogen at room temperature for 2 hours. Allyltributyl stannane ( $0.093 \mathrm{~mL}, 0.3 \mathrm{mmol}, 0.6$ equiv.) was added to the resulting mixture and stirred for 10 minutes followed by addition of $1 \mathrm{~mL}$ of $h \operatorname{mim}\left[\mathrm{PF}_{6}{ }^{-}\right]$to the pre-formed catalyst. The organic solvent was removed in vacuo and subsequent dropwise addition of 2-bromoacrolein (67.5 $\mathrm{mg}, 0.5 \mathrm{mmol}, 1.0$ equiv.) and cyclopentadiene $(0.10 \mathrm{~mL}, 1.5 \mathrm{mmol}, 3.0$ equiv.) along side of the flask were carried out. The reaction mixture was stirred at room temperature for $20 \mathrm{~h}$. The mixture was extracted with ether $(10 \mathrm{~mL} \mathrm{X} \mathrm{3).} \mathrm{The} \mathrm{combined} \mathrm{organic} \mathrm{extracts} \mathrm{was} \mathrm{washed}$ with brine, dried over anhydrous $\mathrm{MgSO}_{4}$, filtered and concentrated in vacuo. The residual crude product was purified via silica gel chromatography to afford the Diels-Alder adduct as a colourless solid (92\% yield). 


\section{Procedure for Asymmetric Diels-Alder Reaction in Ionic Liquid (recyclability)}

Representative procedure for enantioselective Diels-Alder reaction: Preparation of ( $1 R$ ,2R, 4R) -2-bromo-bicyclo[2.2.1]hept-5-ene-2-carbaldehyde

To an oven dried $10 \mathrm{~mL}$ round-bottom flask equipped with a magnetic stirring bar was added $\mathrm{InCl}_{3}$ (22 mg, $0.1 \mathrm{mmol}, 0.2$ equiv.). The solid was azeotropically dried with anhydrous tetrahydrofuran twice $(2 \mathrm{~mL} \times 2)$ prior to the addition of $1.5 \mathrm{~mL}$ of dichloromethane. (S)BINOL (31 mg, $0.11 \mathrm{mmol}, 0.22$ equiv.) and $4 \AA ̊$ molecular sieve ( $15 \mathrm{mg}$ ) were added and the mixture was stirred under nitrogen at room temperature for 2 hours. Allyltributyl stannane (0.093 mL, $0.3 \mathrm{mmol}, 0.6$ equiv.) was added to the resulting mixture and stirred for 10 minutes followed by addition of $1 \mathrm{~mL}$ of $\operatorname{hmim}\left[\mathrm{PF}_{6}{ }_{6}^{-}\right]$to the pre-formed catalyst. The organic solvent was removed in vacuo and subsequent dropwise addition of 2-bromoacrolein (67.5 $\mathrm{mg}, 0.5 \mathrm{mmol}, 1.0$ equiv.) and cyclopentadiene $(0.10 \mathrm{~mL}, 1.5 \mathrm{mmol}, 3.0$ equiv.) along side of the flask were carried out. The reaction mixture was stirred at room temperature for $20 \mathrm{~h}$. The rubber septum was removed and the mixture was extracted with hexane $(10 \mathrm{~mL} \mathrm{X} \mathrm{5).} \mathrm{The}$ round- bottom flask was resealed with a rubber septum and anhydrous THF ( $2 \mathrm{~mL} X 2)$ was added to the hmim[ $\left[\mathrm{PF}_{6}^{-}\right]$containing chiral In(III) complex mixture for the removal of residual hexane and moisture in vacuo. The mixture was purge with nitrogen prior to addition of 2bromoacrolein ( $67.5 \mathrm{mg}, 0.5 \mathrm{mmol}, 1.0$ equiv.) and cyclopentadiene $(0.10 \mathrm{~mL}, 1.5 \mathrm{mmol}, 3.0$ equiv.) for subsequent cycles of Diels-Alder reaction. The combined organic extracts was washed with brine, dried over anhydrous $\mathrm{MgSO}_{4}$, filtered and concentrated in vacuo. The residual crude product was purified via flash column chromatography to afford the DielsAlder adduct as a colourless solid. 


\section{Characterization of Diels-Alder adduct}

$(1 R, 2 R, 4 R)$-2-Bromo-bicyclo[2.2.1]hept-5-ene-2-carbaldehyde

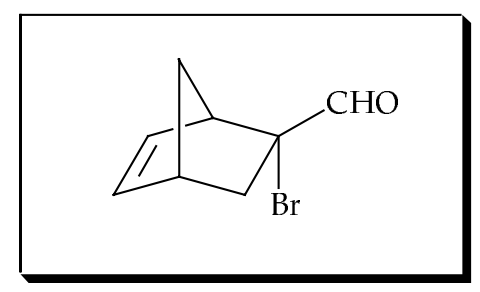

$(98 \% e e)$

exo : endo 97 : 3

Colorless oil (92 mg, 92\%); $\mathrm{R}_{\mathrm{f}}=0.65$ (4:1 hexane/ethyl acetate)

${ }^{1} \mathrm{H}$ NMR (300 MHz, $\left.\mathrm{CDCl}_{3}\right): \delta 9.54(\mathrm{~s}, 1 \mathrm{H}$, exo $), 6.45(\mathrm{dd}, J=3.1,5.6 \mathrm{~Hz}, 1 \mathrm{H}), 6.14(\mathrm{dd}, J=$ 3.1, $5.6 \mathrm{~Hz}, 1 \mathrm{H}), 3.25$ (bs, 1H), 2.97 (bs, 1H), 2.65 (dd, J = 3.5, $13.6 \mathrm{~Hz}, 1 \mathrm{H}), 1.42-1.59$ (m, $2 \mathrm{H}), 1.32(\mathrm{~d}, J=9.4 \mathrm{~Hz}, 1 \mathrm{H})$.

${ }^{13} \mathrm{C} \mathrm{NMR}\left(75.4 \mathrm{MHz}, \mathrm{CDCl}_{3}\right): \delta 191.9,140.0,133.8,72.6,49.6,46.7,42.4,36.9$.

FTIR (neat): 2978, $1722 \mathrm{~cm}^{-1}$.

HRMS Calcd for $\mathrm{C}_{8} \mathrm{H}_{19} \mathrm{BrO}[\mathrm{M}]^{+}$: 199.9837. Found:199.9834

$[\alpha]_{\mathrm{D}}=+9.6^{\mathrm{o}}\left(\mathrm{c}=1.37 \mathrm{~g} / 100 \mathrm{~mL}, \mathrm{CH}_{2} \mathrm{Cl}_{2}\right)$

Diastereoselectivity (exo-endo ratio) was determined by ${ }^{1} \mathrm{H}$ NMR analysis of the crude mixture: $\delta 9.56$ (s, 1H, exo, major), 9.34 (s, 1H, endo, minor). Enantioselectivity was determined by reduction with $\mathrm{NaBH}_{4}$ to the corresponding alcohol, conversion to the $(R)$ MTPA ester derivative and ${ }^{1} \mathrm{H}$ NMR integration (500 MHz, $\left.\mathrm{CDCl}_{3}\right): \delta 4.74(\mathrm{~d}, 1 \mathrm{H}$, minor), 4.67 (d, 1H, major), 4.61 (d,1H, major), 4.52 (d, 1H, minor).

The absolute configuration was assigned by measurement of optical rotation and comparison with known substances. ${ }^{4}$

${ }^{4}$ Corey, E. J.; Shibata, T.; Lee, T. W. J. Am. Chem. Soc. 2002, 124, 3808. 


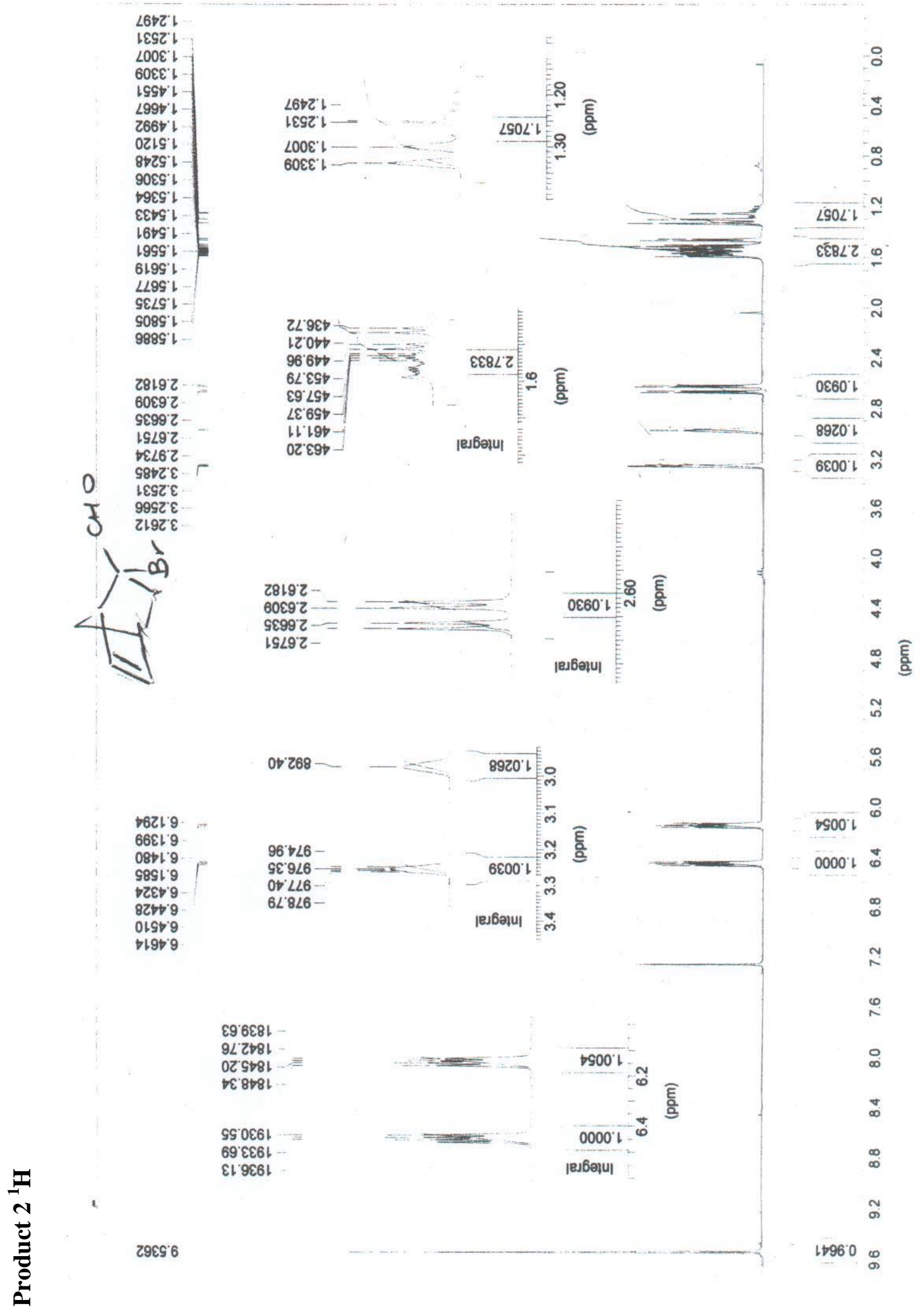




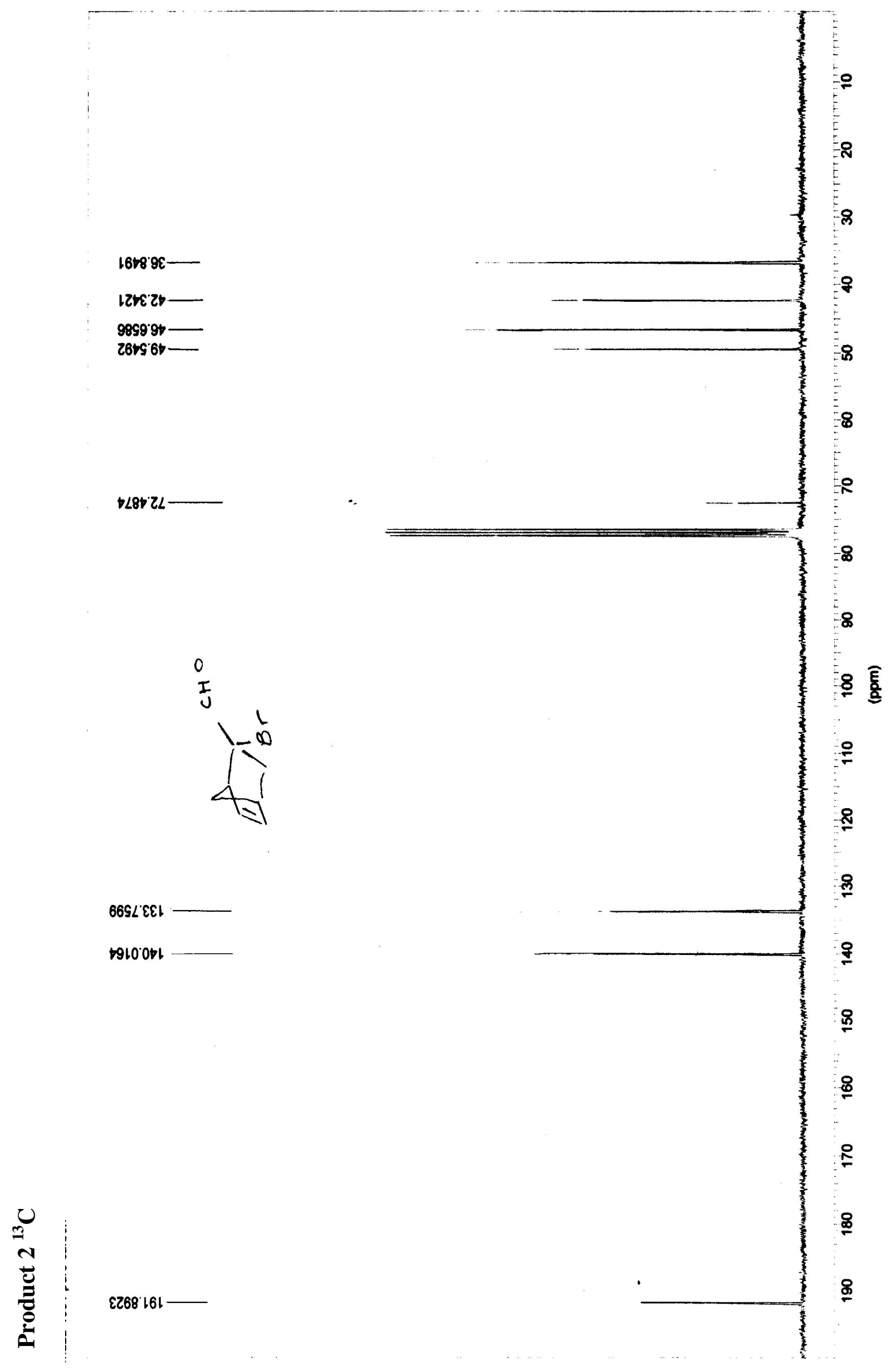




\section{(1R, 2S, 4R)-2-Methyl-bicyclo[2.2.1]hept-5-ene-2-carbaldehyde}

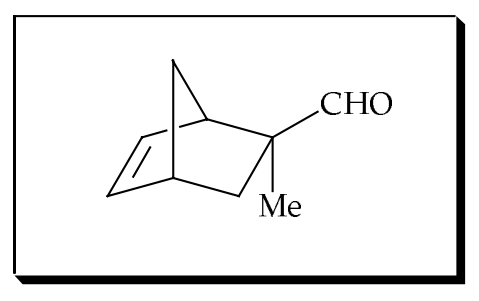

$(98 \%$ \%e $)$

exo : endo $97: 3$

Colorless oil (60mg, 89\%); $\mathrm{R}_{\mathrm{f}}=0.64$ (4:1 hexane/ethyl acetate)

${ }^{1} \mathrm{H}$ NMR $\left(300 \mathrm{MHz}, \mathrm{CDCl}_{3}\right) \delta 9.68(\mathrm{~s}, 1 \mathrm{H}), 6.28(\mathrm{dd}, J=3.1,5.6 \mathrm{~Hz}, 1 \mathrm{H}), 6.09(\mathrm{dd}, J=3.1$, $5.6 \mathrm{~Hz}, 1 \mathrm{H}), 2.88$ (bs, 1H), $2.80(\mathrm{bs}, 1 \mathrm{H}), 2.24(\mathrm{dd}, J=3.8,11.9 \mathrm{~Hz}, 1 \mathrm{H}), 1.38-1.37$ (m, 2H), $1.00(\mathrm{~s}, 3 \mathrm{H}), 0.75(\mathrm{bd}, J=11.8 \mathrm{~Hz}, 1 \mathrm{H})$.

${ }^{13} \mathrm{C}$ NMR $\left(100 \mathrm{MHz}, \mathrm{CDCl}_{3}\right): \delta 205.7,139.5,133.0,53.8,48.3,47.5,43.1,34.5,19.9$

FTIR (neat): $2918,1726 \mathrm{~cm}^{-1}$.

HRMS Calcd for $\mathrm{C}_{9} \mathrm{H}_{12} \mathrm{O}[\mathrm{M}]^{+}:$136.0888. Found: 136.0895 .

$[\alpha]_{\mathrm{D}}=+13.5^{\mathrm{o}}\left(\mathrm{c}=3.10 \mathrm{~g} / 100 \mathrm{~mL}, \mathrm{CH}_{2} \mathrm{Cl}_{2}\right)$

Diastereoselectivity (exo-endo ratio) was determined by ${ }^{1} \mathrm{H}$ NMR analysis of the crude mixture: $\delta 9.68$ (s, 1H, exo, major), 9.38 (s, 1H, endo, minor). Enantioselectivity was determined by reduction with $\mathrm{NaBH}_{4}$ to the corresponding alcohol, conversion to the $(R)$ MTPA ester derivative and ${ }^{1} \mathrm{H}$ NMR integration (500 MHz, $\mathrm{CDCl} 3$ ): $\delta 4.34$ (d, $1 \mathrm{H}$, major), 4.31 (d, 1H minor), 4.25 (d, 1H, minor), 4.22 (d, 1H, major).

The absolute configuration was assigned by measurement of optical rotation and comparison with known substances. ${ }^{4}$ 


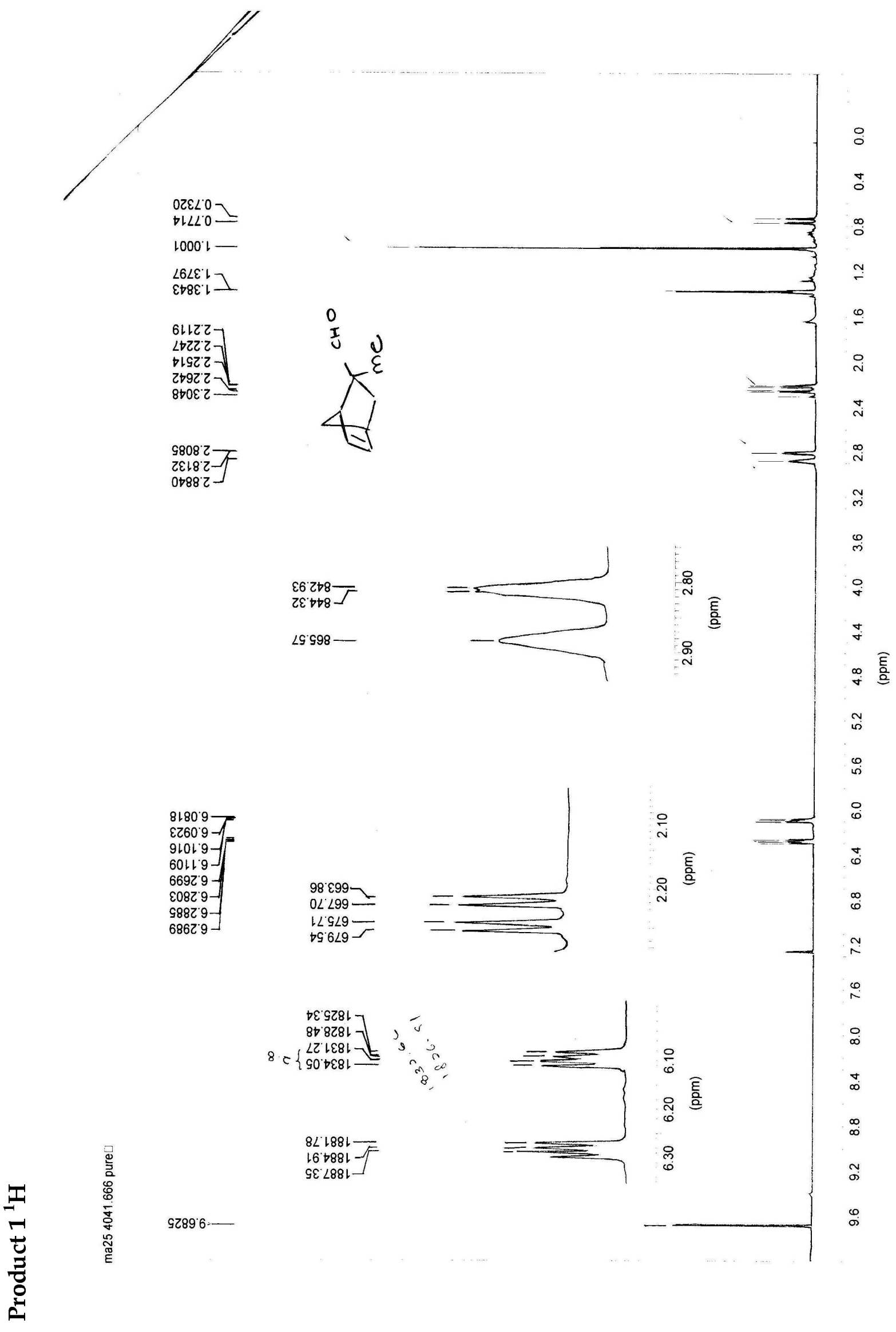




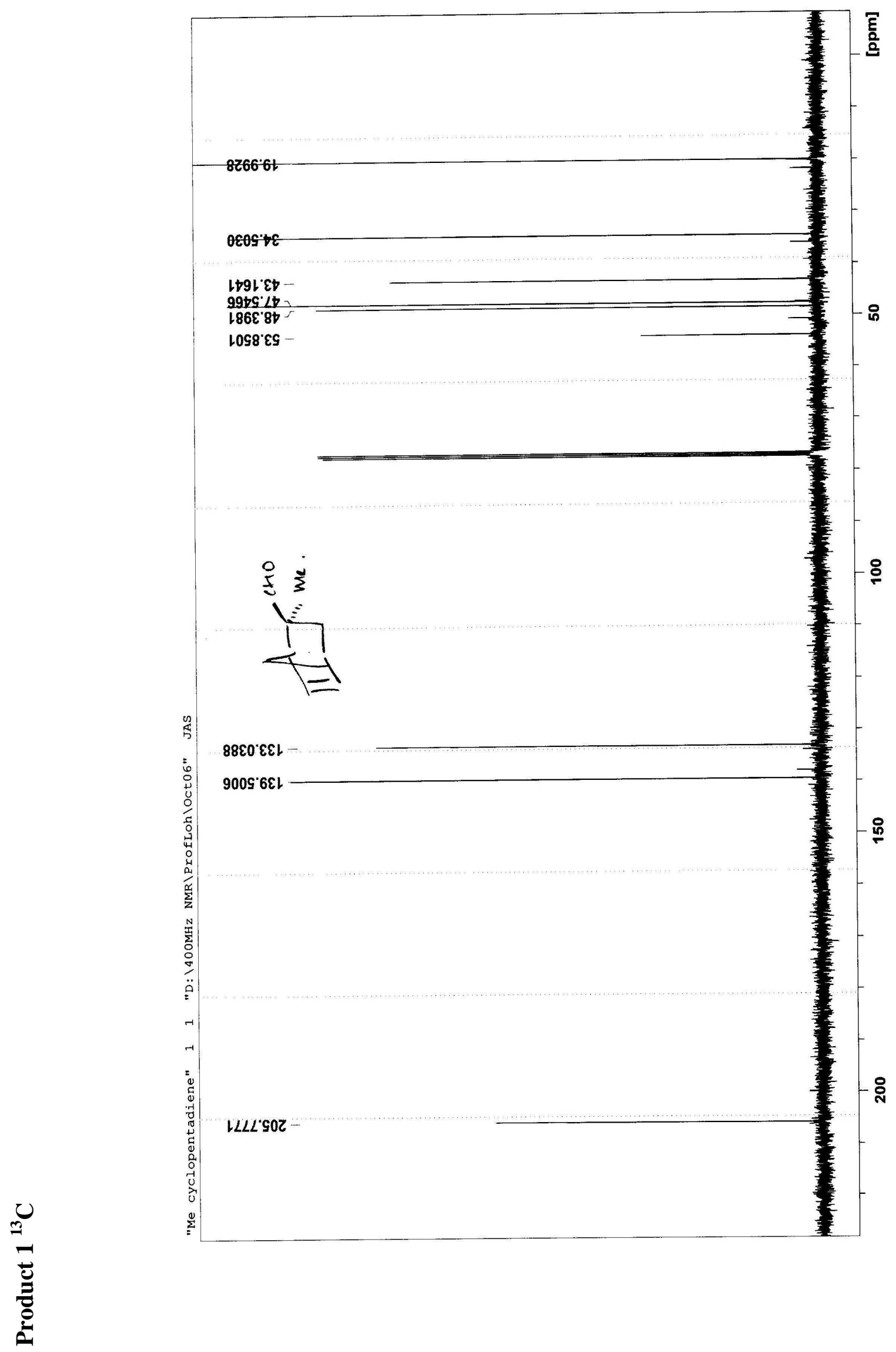




\section{(R)-1-Bromo-4-methyl-cyclohex-3-enecarbaldehyde}

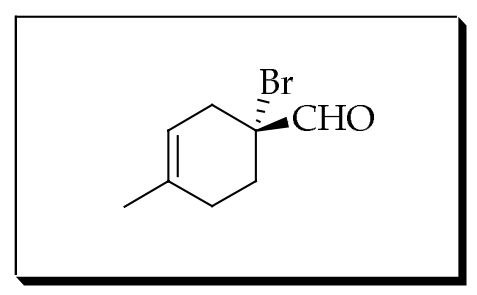

$(96 \% e e)$

Colorless oil (55 mg, 90\%); $\mathrm{R}_{\mathrm{f}}=0.67$ (4:1 hexane/ethyl acetate)

${ }^{1} \mathrm{H}$ NMR (300 MHz, $\mathrm{CDCl}_{3}$ ) $\delta 9.36$ (s, 1H), 5.33 (bs, 1H), 2.79 (bd, 1H, J=18.1 Hz), 2.62

(bd, 1H, $J=18.0 \mathrm{~Hz}$ ), 2.09-2.28 (m, 4H), 1.67 (bs, 3H).

${ }^{13} \mathrm{C}$ NMR $\left(75.4 \mathrm{MHz}, \mathrm{CDCl}_{3}\right): \delta 192.2,134.0,117.0,67.0,34.4,30.9,28.5,23.1$.

FTIR (neat): $2916,1726,1638 \mathrm{~cm}^{-1}$.

HRMS Calcd for $\mathrm{C}_{8} \mathrm{H}_{11} \mathrm{O}$ [M-Br]: 123.0810. Found: 123.0810

$[\alpha]_{\mathrm{D}}=+67.7^{\circ}\left(\mathrm{c}=1.50 \mathrm{~g} / 100 \mathrm{~mL}, \mathrm{CH}_{2} \mathrm{Cl}_{2}\right)$

Enantioselectivity was determined by reduction with $\mathrm{NaBH}_{4}$ to the corresponding alcohol, conversion to the benzoyl ester derivative and HPLC analysis using Daicel Chiral ADH and Daicel Chiral AD column in series with $1.0 \% i$-PrOH in hexanes for elution; $1.0 \mathrm{~mL} / \mathrm{min} ; 235$ $\mathrm{nm}$; retention times: $88.612 \mathrm{~min}$ (minor), 92.248 min (major). ${ }^{5}$

The absolute configuration was assigned by measurement of optical rotation and comparison with known substances. ${ }^{4}$

${ }^{5}$ The Diels-Alder adduct contains ca. 16\% of its regioisomer (1-bromo-3-methylcyclohex-3ene-1-carboxaldehyde) that cannot be separated by column chromatography. 


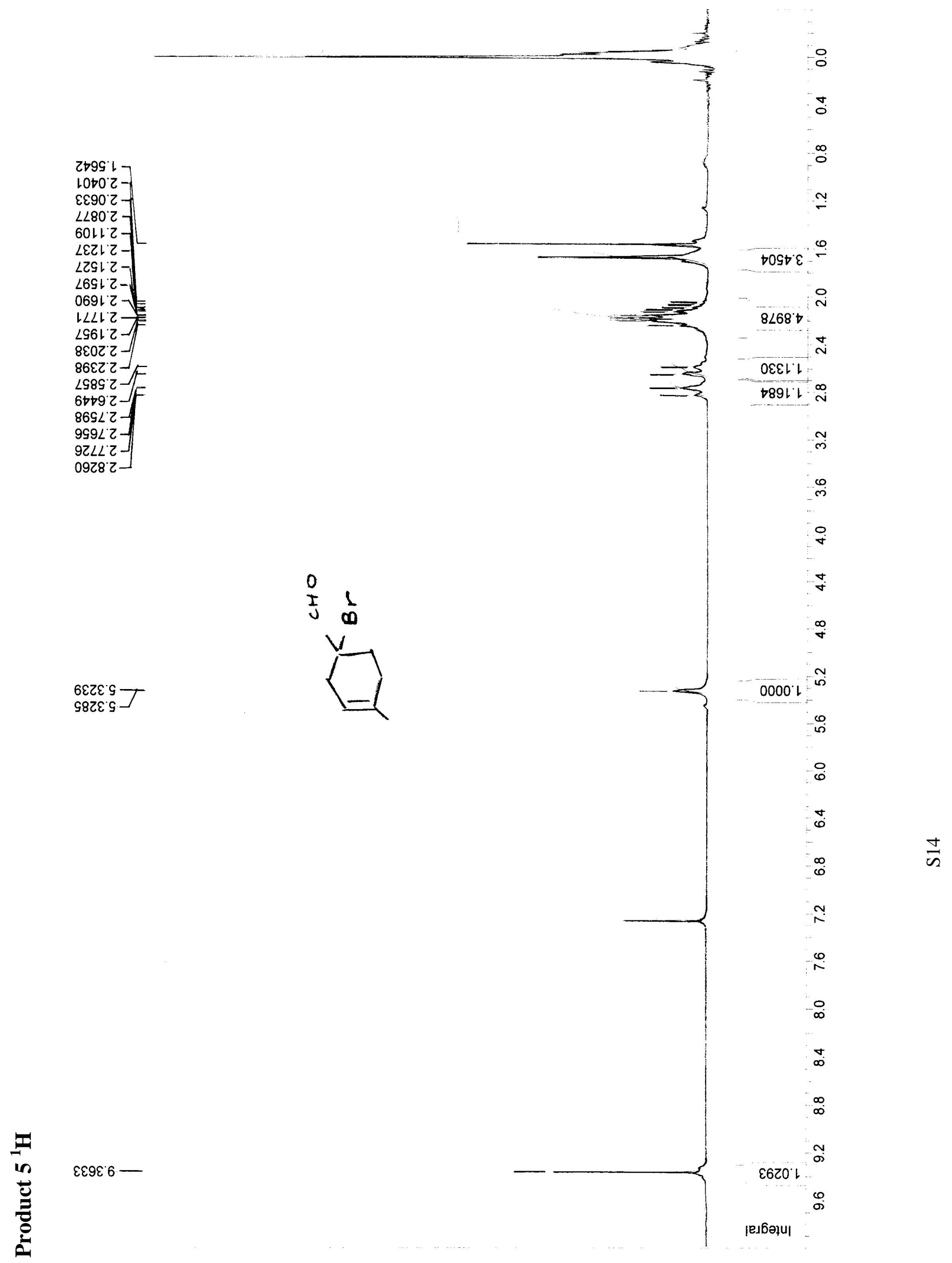



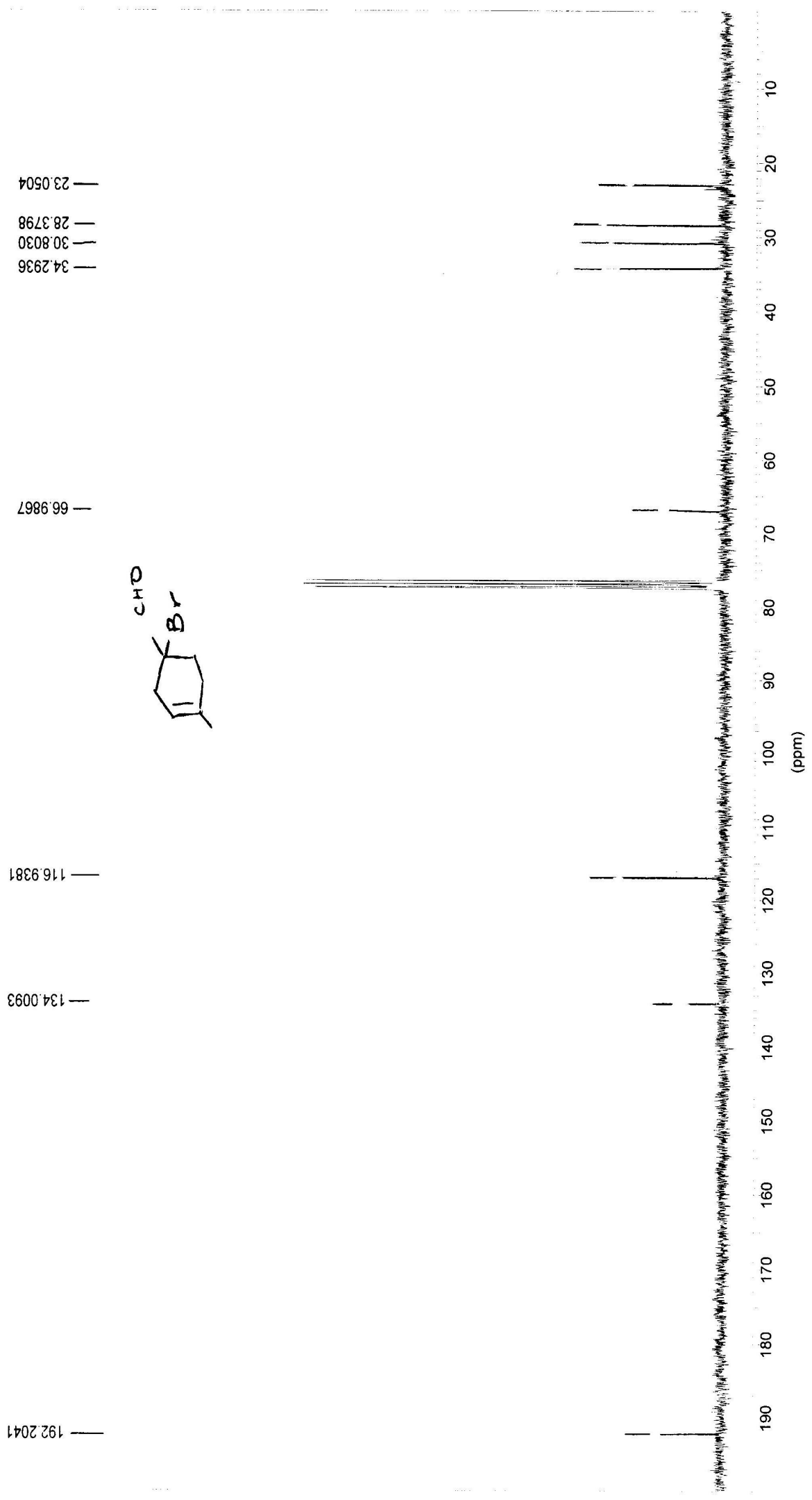


\section{Product 5 HPLC spectrum}

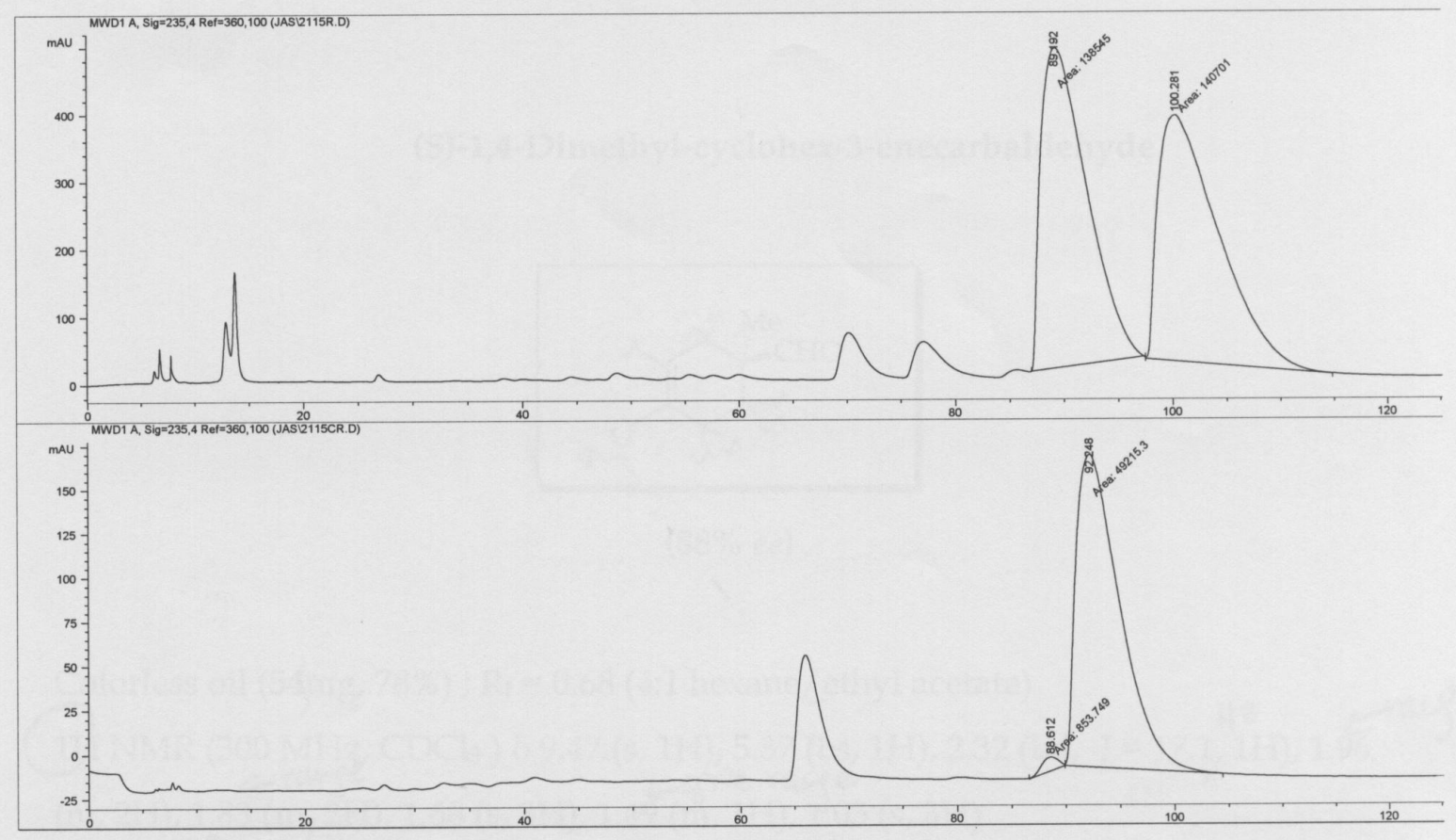

\# Time Area Height Width Area\% Symmetry

$\begin{array}{lllllll}1 & 88.612 & 796.7 & 7.3 & 1.8119 & 1.607 & 1.8\end{array}$

$\begin{array}{llllll}2 & 92.248 & 48780.7176 .6 & 4.6043 & 98.393 & 0.457\end{array}$

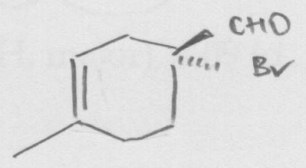




\section{(S)-1,4-Dimethyl-cyclohex-3-enecarbaldehyde}

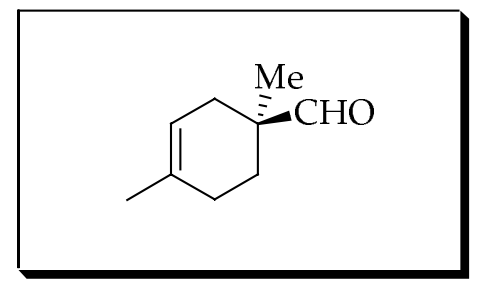

$(88 \% e e)$

Colorless oil (54mg, 78\%); $\mathrm{R}_{\mathrm{f}}=0.68$ (4:1 hexane/ethyl acetate)

${ }^{1} \mathrm{H}$ NMR $\left(300 \mathrm{MHz}, \mathrm{CDCl}_{3}\right) \delta 9.47(\mathrm{~s}, 1 \mathrm{H}), 5.37(\mathrm{bs}, 1 \mathrm{H}), 2.32(\mathrm{bd}, J=17.1 \mathrm{~Hz}, 1 \mathrm{H}), 1.96$ (m, 2H), 1.83 (m. 2H), 1.68 (s, 3H), 1.49 (m, 1H), 1.03 (s, 3H)

${ }^{13} \mathrm{C}$ NMR $\left(100 \mathrm{MHz}, \mathrm{CDCl}_{3}\right)$ : 206.1, 133.7, 118.3, 44.3, 31.7, 29.0, 26.8, 23.4, 20.7

FTIR (neat): 2924, 1725, $1633 \mathrm{~cm}^{-1}$.

$[\alpha]_{\mathrm{D}}=+42.0^{\circ}\left(\mathrm{c}=3.28 \mathrm{~g} / 100 \mathrm{~mL}, \mathrm{CH}_{2} \mathrm{Cl}_{2}\right)$

Enantioselectivity was determined by reduction with $\mathrm{NaBH}_{4}$ to the corresponding alcohol, conversion to the $(R)$-MTPA ester derivative and ${ }^{1} \mathrm{H}$ NMR integration $(500 \mathrm{MHz}, \mathrm{CDCl} 3): \delta$ 4.14 (d, 1H, major), 4.09 (d, 1H minor), 4.03 (d, 1H, minor), 3.98 (d, 1H, major).

The absolute configuration was assigned by measurement of optical rotation and comparison with known substances ${ }^{4}$ and by analogy with $(R)$-1-bromo-4-methyl-cyclohex-3enecarbaldehyde.

${ }^{5}$ The Diels-Alder adduct contains ca. $23 \%$ of its regioisomer that cannot be separated by column chromatography. 


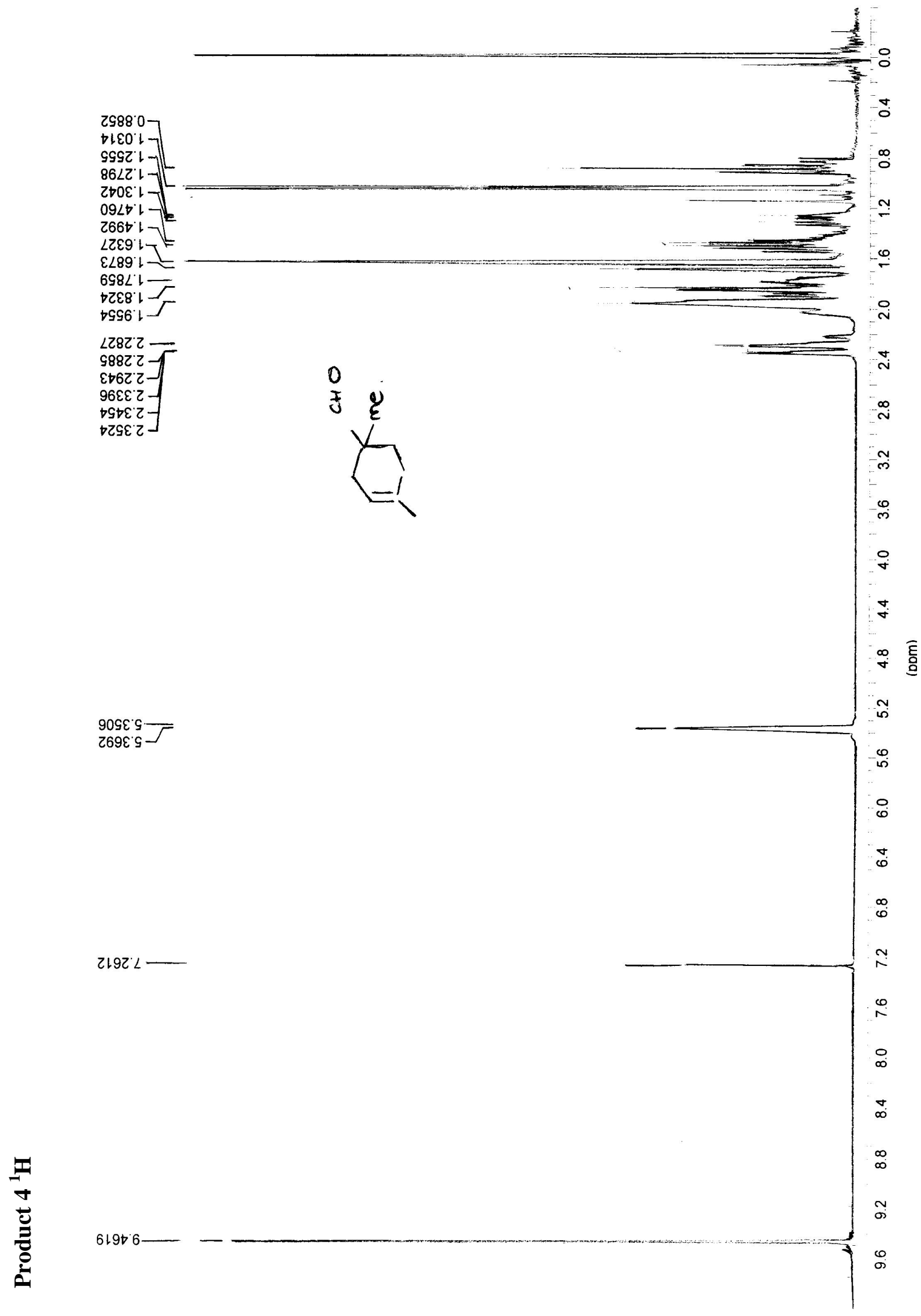




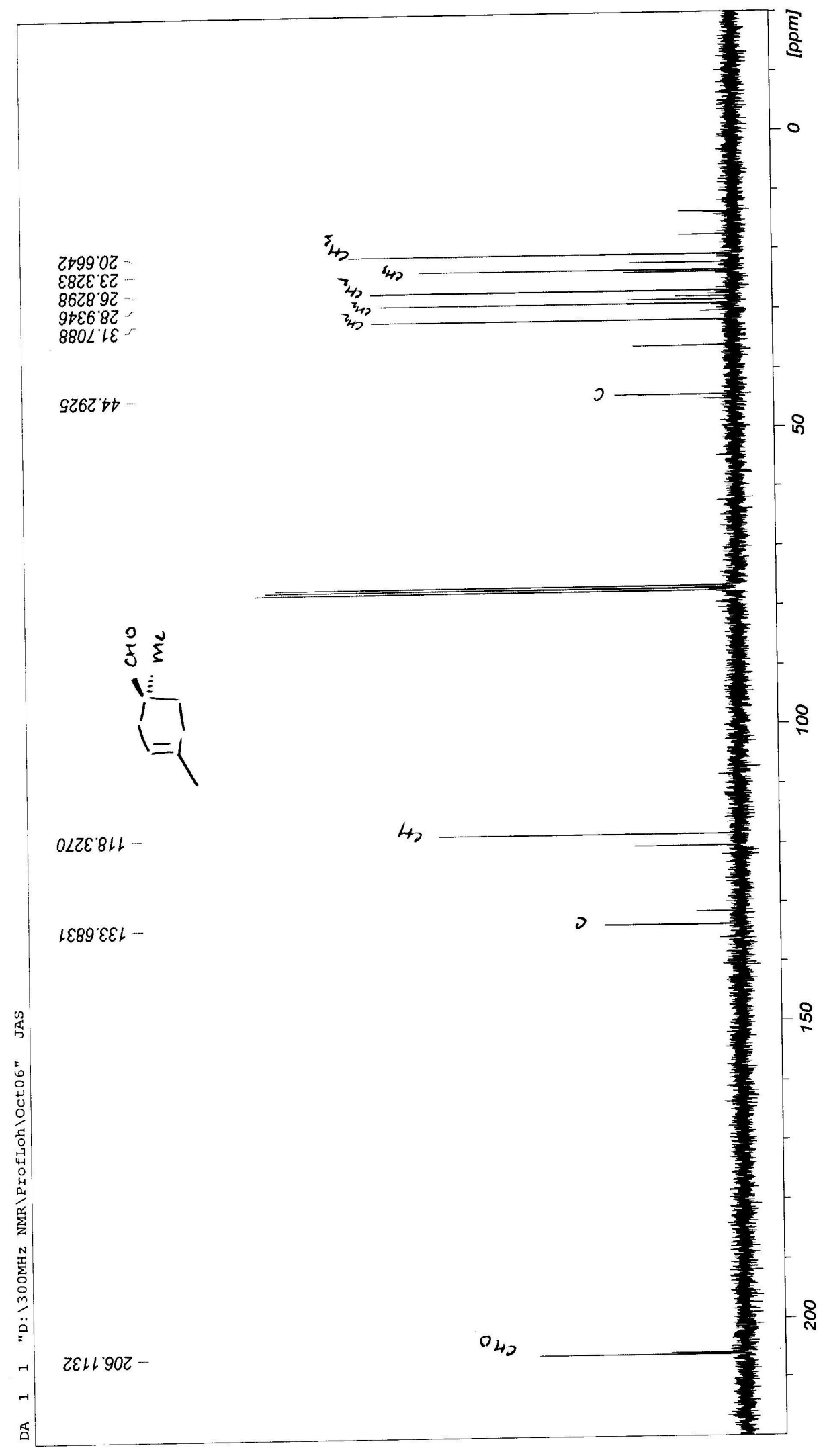

$\frac{a}{n}$

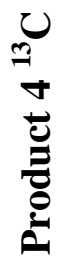




\section{(R)-1-Bromo-3,4-dimethyl-cyclohex-3-enecarbaldehyde}

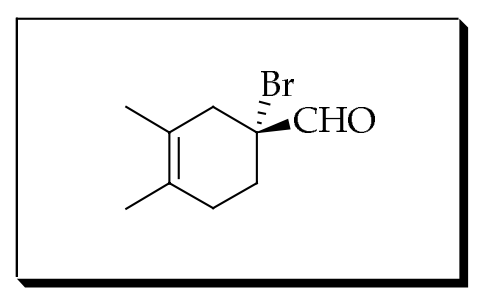

$(94 \% e e)$

Colorless oil (97 mg, 90\%); $\mathrm{R}_{\mathrm{f}}=0.67$ (4:1 hexane/ethyl acetate)

${ }^{1} \mathrm{H}$ NMR (300 MHz, $\left.\mathrm{CDCl}_{3}\right) \delta 9.34(\mathrm{~s}, 1 \mathrm{H}), 2.74(\mathrm{bd}, J=17.4 \mathrm{~Hz}, 1 \mathrm{H}), 2.56(\mathrm{bd}, J=17.8$ $\mathrm{Hz}, 1 \mathrm{H}), 2.08-2.27$ (m, 4H), 1.65 (s, 3H), 1.62 (s, 3H)

${ }^{13} \mathrm{C}$ NMR $\left(75.4 \mathrm{MHz}, \mathrm{CDCl}_{3}\right): \delta 192.2,125.4,122.2,67.7,40.0,31.2,29.9,19.0,18.6$.

FTIR (neat): $2916,1726,1641 \mathrm{~cm}^{-1}$.

HRMS Calcd for $\mathrm{C}_{19} \mathrm{H}_{13} \mathrm{BrO}[\mathrm{M}]^{+}: 216.0150$. Found: 216.0141 .

$[\alpha]_{\mathrm{D}}=+62.3^{\mathrm{o}}\left(\mathrm{c}=3.19 \mathrm{~g} / 100 \mathrm{~mL}, \mathrm{CH}_{2} \mathrm{Cl}_{2}\right)$

Enantioselectivity was determined by reduction with $\mathrm{NaBH}_{4}$ to the corresponding alcohol, conversion to the benzoyl ester derivative and HPLC analysis using Daicel Chiral AD column with $1.0 \% i$-PrOH in hexanes for elution; $0.3 \mathrm{~mL} / \mathrm{min} ; 235 \mathrm{~nm}$; retention times: $89.943 \mathrm{~min}$ (minor), $100.579 \mathrm{~min}$ (major).

The absolute configuration was assigned by analogy with $(R)$-1-bromo-4-methyl-cyclohex-3enecarbaldehyde. 


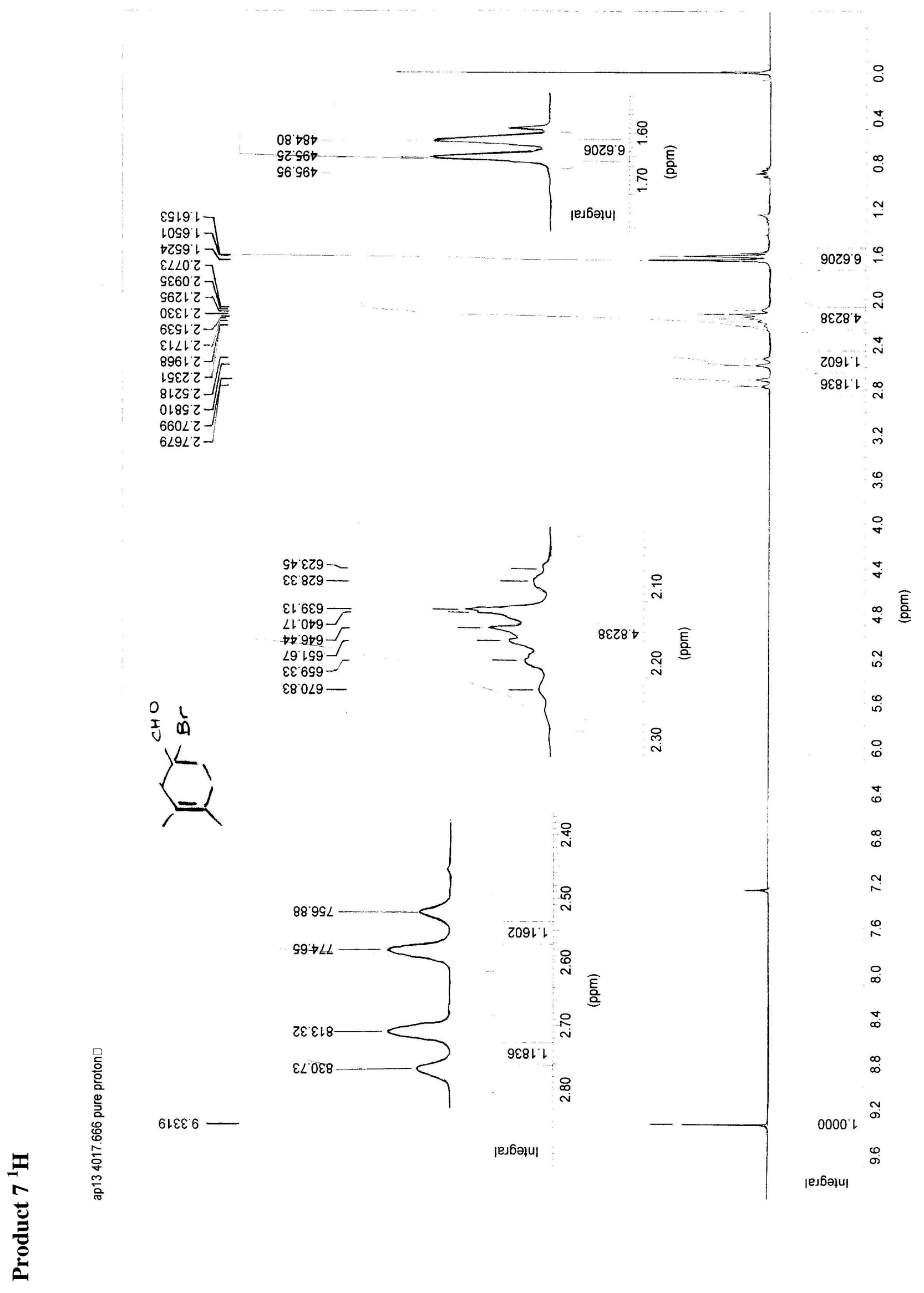


ع609 $81-7$

IZ96 81

$690662=$

$8 \nabla 81 \cdot 1 \varepsilon-$

$20966 \varepsilon$

$16 L L<9$

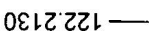

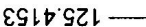

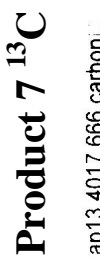

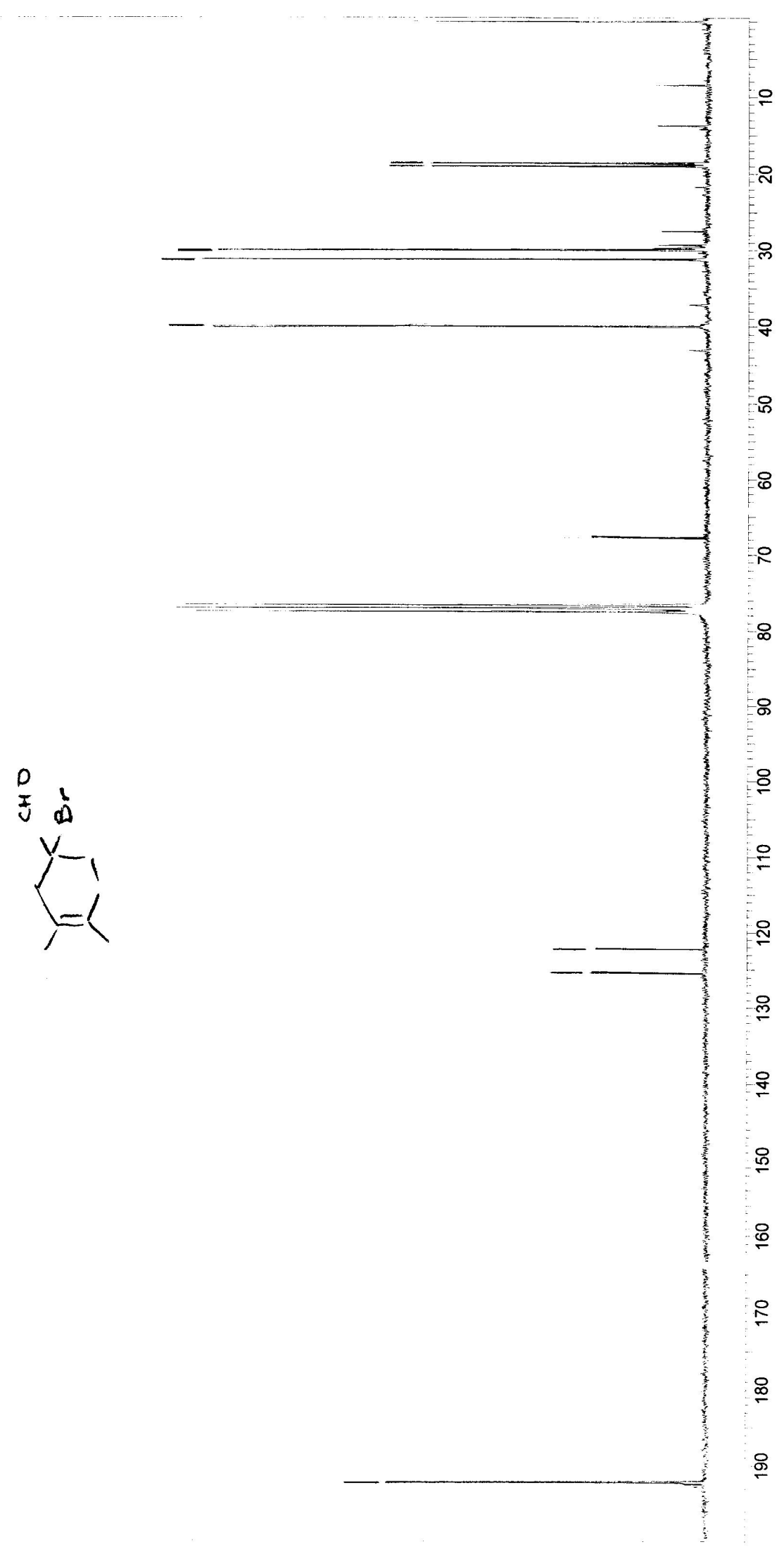




\section{Product 7 HPLC spectrum}
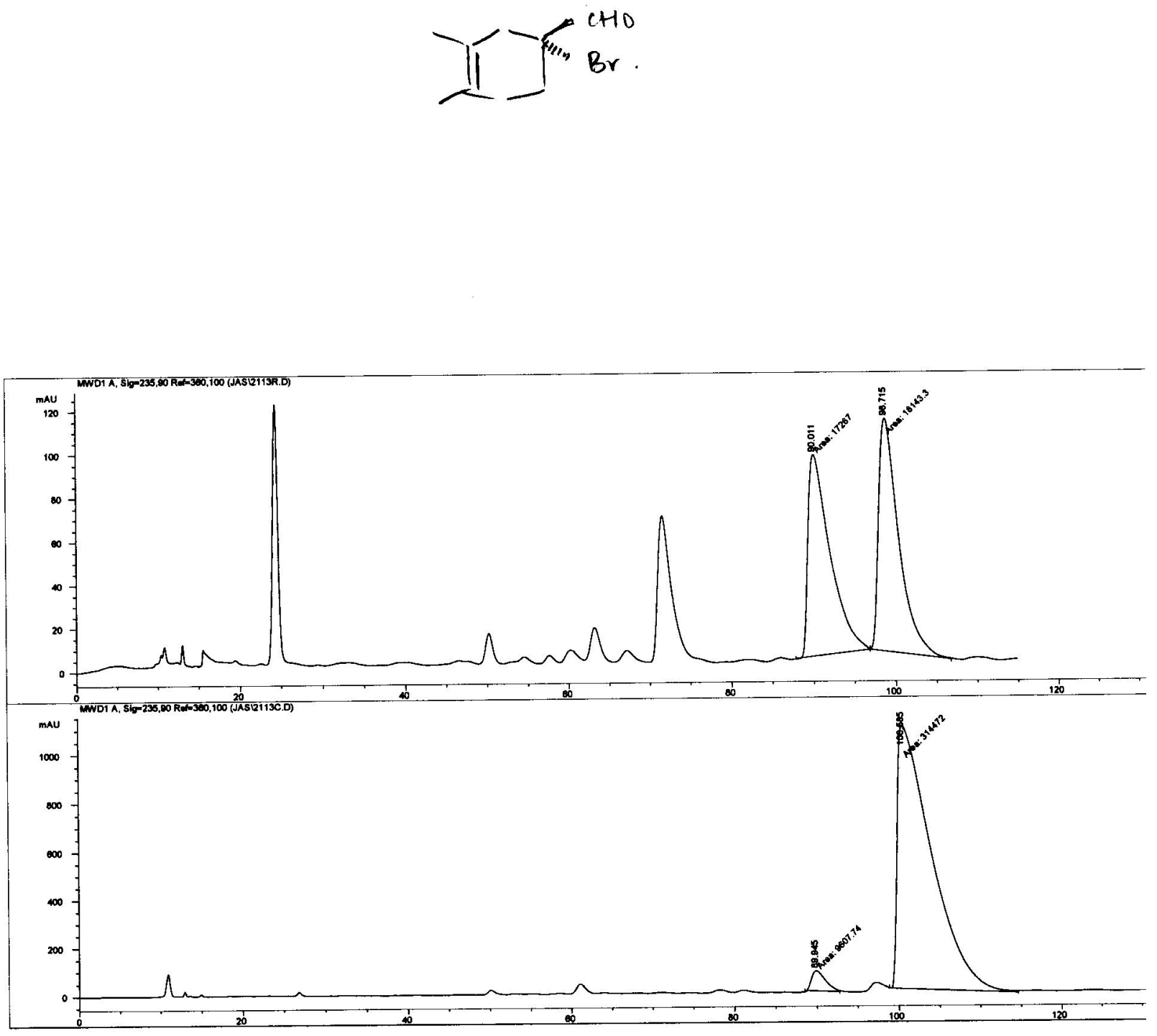

$\begin{array}{llllll}\# & \text { Time } & \text { Area } & \text { Height } & \text { Width } & \text { Area\% } \\ 1 & 89.943 & 9100.7 & 81.2 & 1.867 & 2.831 \\ 2 & 100.579 & 312404.7 & 1087.7 & 4.787 & 97.169\end{array}$




\section{(S)-1,3,4-Trimethyl-cyclohex-3-enecarbaldehyde}

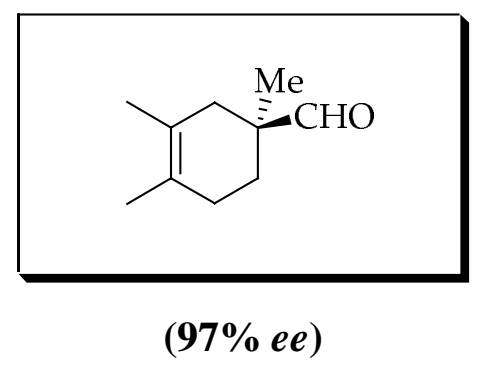

Colorless oil (67 mg, 88\%); $\mathrm{R}_{\mathrm{f}}=0.68$ (4:1 hexane/ethyl acetate)

${ }^{1} \mathrm{H}$ NMR (300 MHz, $\left.\mathrm{CDCl}_{3}\right) \delta 9.45(\mathrm{~s}, 1 \mathrm{H}), 2.25(\mathrm{bd}, J=17.4 \mathrm{~Hz}, 1 \mathrm{H}), 1.97(\mathrm{bs}, 2 \mathrm{H}), 1.73-$ 1.85 (m. 2H), $1.64(\mathrm{~s}, 3 \mathrm{H}), 1.59(\mathrm{~s}, 3 \mathrm{H}), 1.41-1.50(\mathrm{~m}, 1 \mathrm{H}), 1.02(\mathrm{~s}, 3 \mathrm{H})$

${ }^{13} \mathrm{C}$ NMR $\left(75.4 \mathrm{MHz}, \mathrm{CDCl}_{3}\right): \delta 206.2,125.2,123.1,45.3,38.0,29.3,28.5,20.7,19.2,18.8$. FTIR (neat): $2918,1726 \mathrm{~cm}^{-1}$.

HRMS Calcd for $\mathrm{C}_{10} \mathrm{H}_{16} \mathrm{O}[\mathrm{M}]^{+}:$152.1201. Found: 152.1198 .

$[\alpha]_{\mathrm{D}}=+48.0^{\circ}\left(\mathrm{c}=4.60 \mathrm{~g} / 100 \mathrm{~mL}, \mathrm{CH}_{2} \mathrm{Cl}_{2}\right)$

Enantioselectivity was determined by reduction with $\mathrm{NaBH}_{4}$ to the corresponding alcohol, conversion to the $(R)-M T P A$ ester derivative and ${ }^{1} \mathrm{H}$ NMR integration (500 MHz, $\mathrm{CDCl} 3$ ): $\delta$ 4.12 (d, 1H, major), 4.08 (d, 1H minor), 4.01 (d, 1H, minor), 3.98 (d, 1H, major).

The absolute configuration was assigned by analogy with $(R)$-1-bromo-4-methyl-cyclohex-3enecarbaldehyde. 


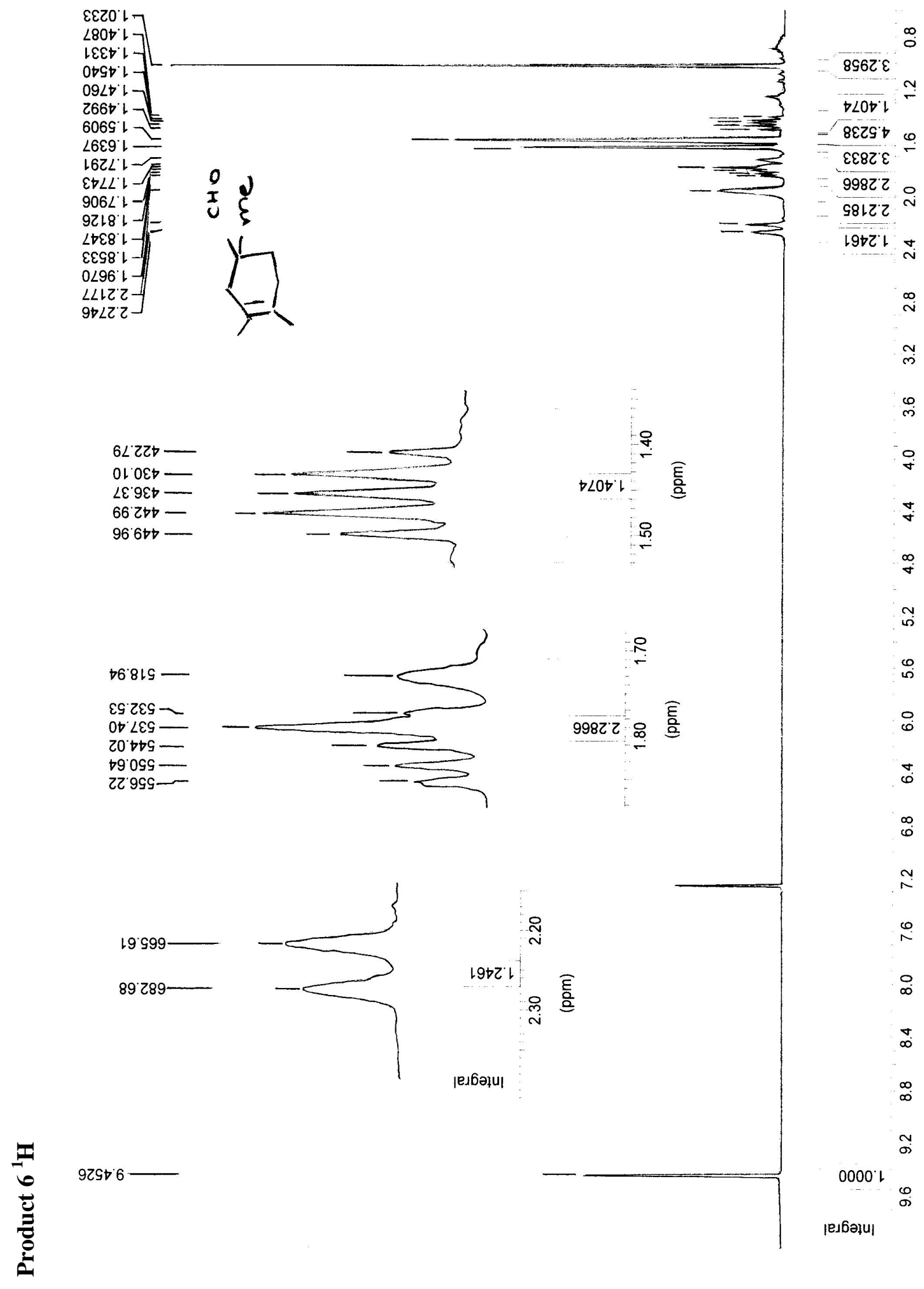



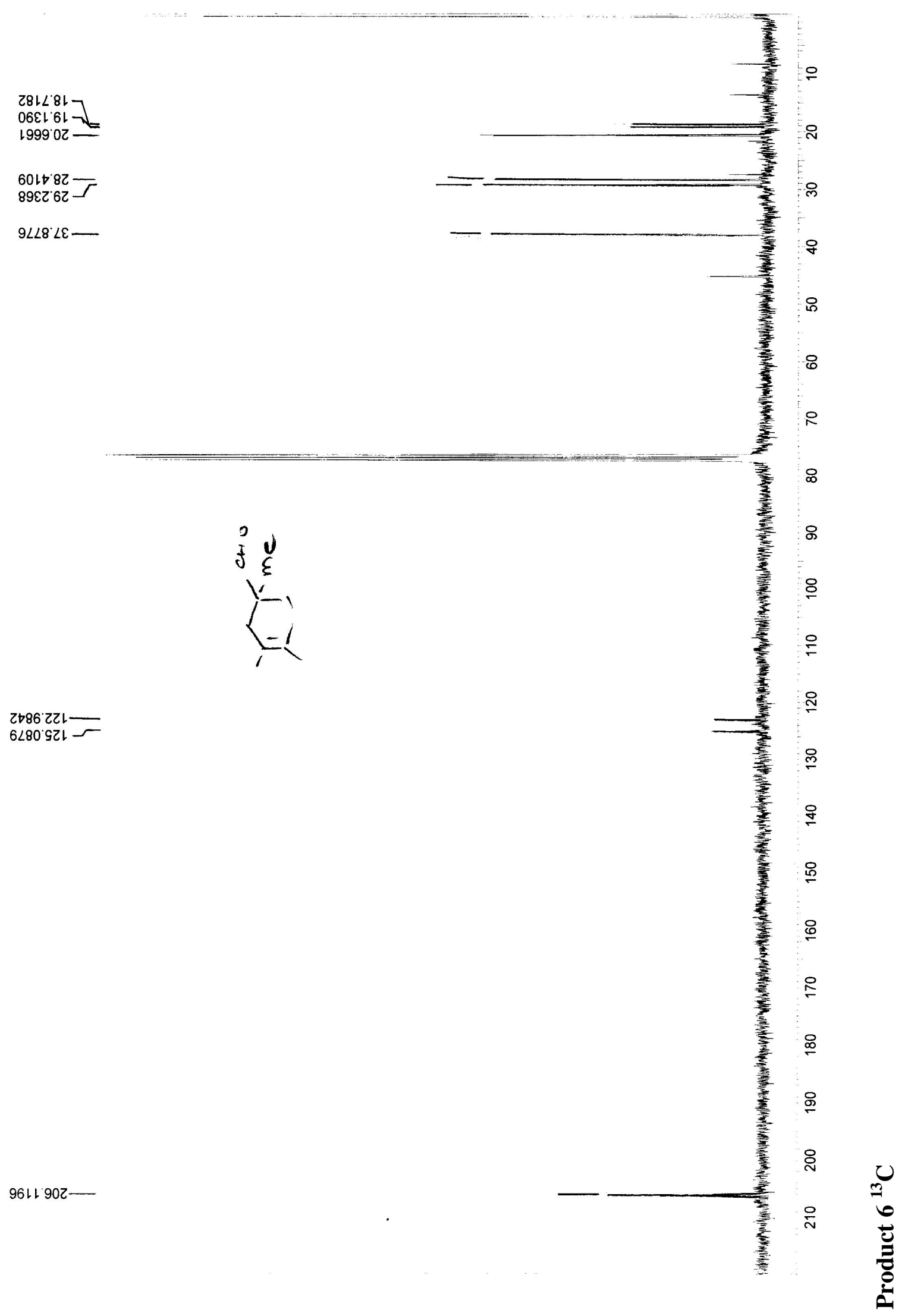


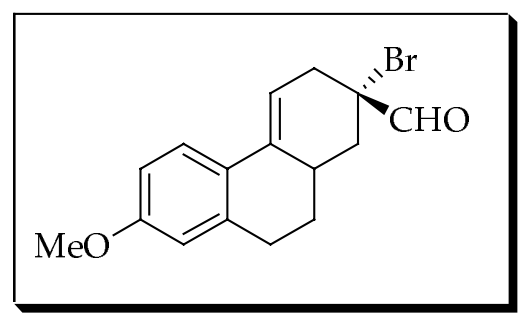

$(92 \%$ ee $)$

Light yellow solid (142mg, 89\%); $\mathrm{R}_{\mathrm{f}}=0.51$ (4:1 hexane/ethyl acetate)

${ }^{1} \mathrm{H}$ NMR $\left(300 \mathrm{MHz}, \mathrm{CDCl}_{3}\right) \delta 9.52(\mathrm{~s}, 1 \mathrm{H}), 7.56(\mathrm{~d}, J=8.7,1 \mathrm{H}), 6.75(\mathrm{dd}, J=8.7,2.8,1 \mathrm{H})$, $6.63(\mathrm{~d}, J=2.4,1 \mathrm{H}), 6.09-6.11(\mathrm{~m}, 1 \mathrm{H}), 3.80(\mathrm{~s}, 3 \mathrm{H}), 2.93-3.10(\mathrm{~m}, 2 \mathrm{H}), 2.71-2.90(\mathrm{~m}$, $3 \mathrm{H}), 2.02-2.37(\mathrm{~m}, 1 \mathrm{H}), 2.02-2.06(\mathrm{~m}, 1 \mathrm{H}), 1.52-1.68(\mathrm{~m}, 2 \mathrm{H})$

${ }^{13} \mathrm{C}$ NMR $\left(75.4 \mathrm{MHz}, \mathrm{CDCl}_{3}\right): \delta 192.6,158.9,137.9,135.5,126.3,125.2,113.3,112.9$,

$111.9,68.2,55.3,37.2,34.7,33.9,30.2,30.1$

FTIR (neat): 2928, 1717, 1494, 1234, $810 \mathrm{~cm}^{-1}$.

HRMS Calcd for $\mathrm{C}_{16} \mathrm{H}_{17} \mathrm{BrO}_{2}[\mathrm{M}]^{+}: 320.0412$. Found:320.0408

$[\alpha]_{\mathrm{D}}=-58.2^{\circ}\left(\mathrm{c}=2.0 \mathrm{~g} / 100 \mathrm{~mL}, \mathrm{CH}_{2} \mathrm{Cl}_{2}\right)$

Enantioselectivity was determined by HPLC analysis using Daicel Chiral ADH column with $1.0 \% i$-PrOH in hexanes for elution; $1.0 \mathrm{~mL} / \mathrm{min} ; 235 \mathrm{~nm}$; retention times: $10.650 \mathrm{~min}$ (major), $12.492 \mathrm{~min}$ (minor).

The absolute configuration was assigned by analogy with $(R)$-1-bromo-4-methyl-cyclohex-3enecarbaldehyde. 


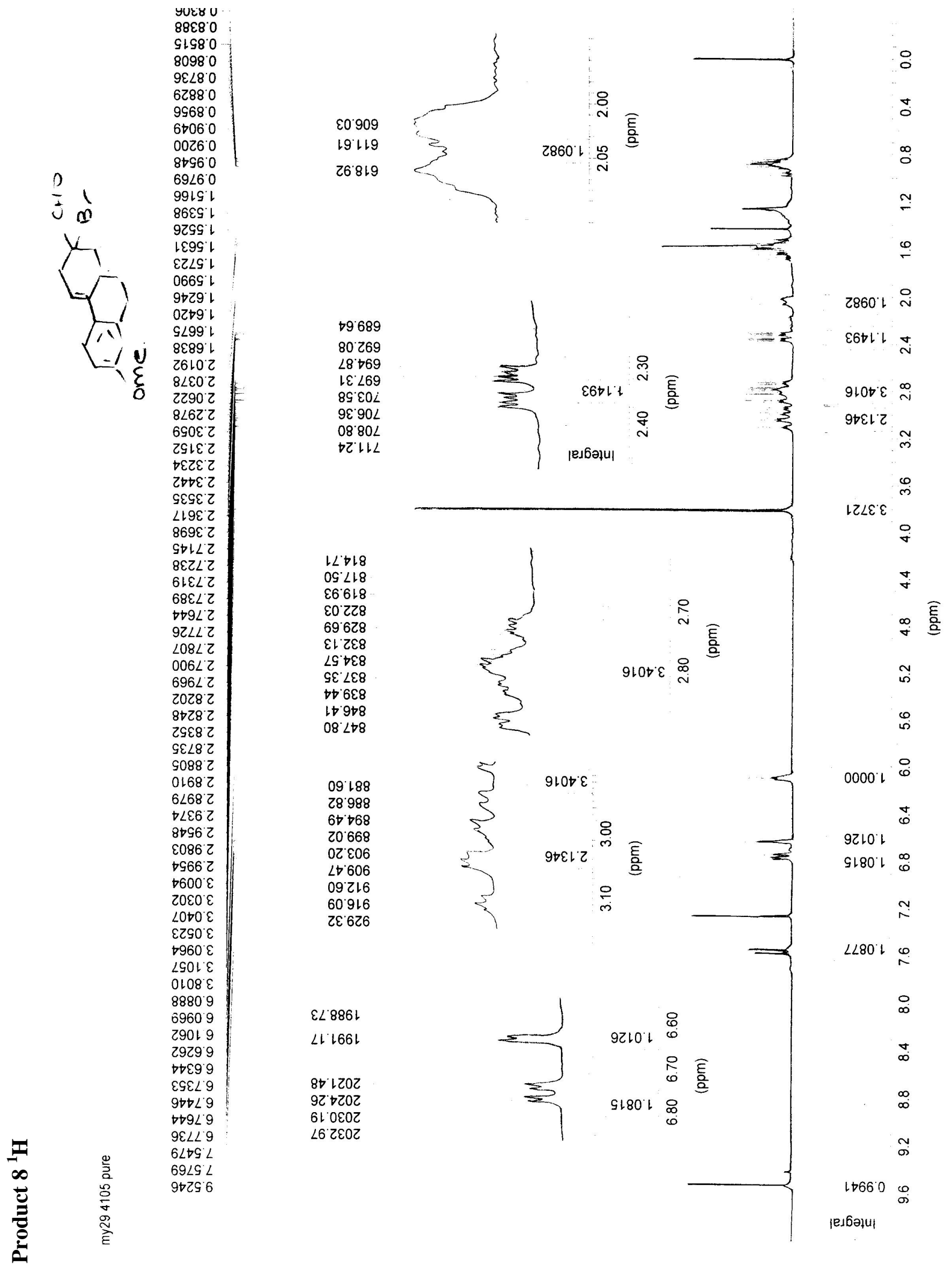




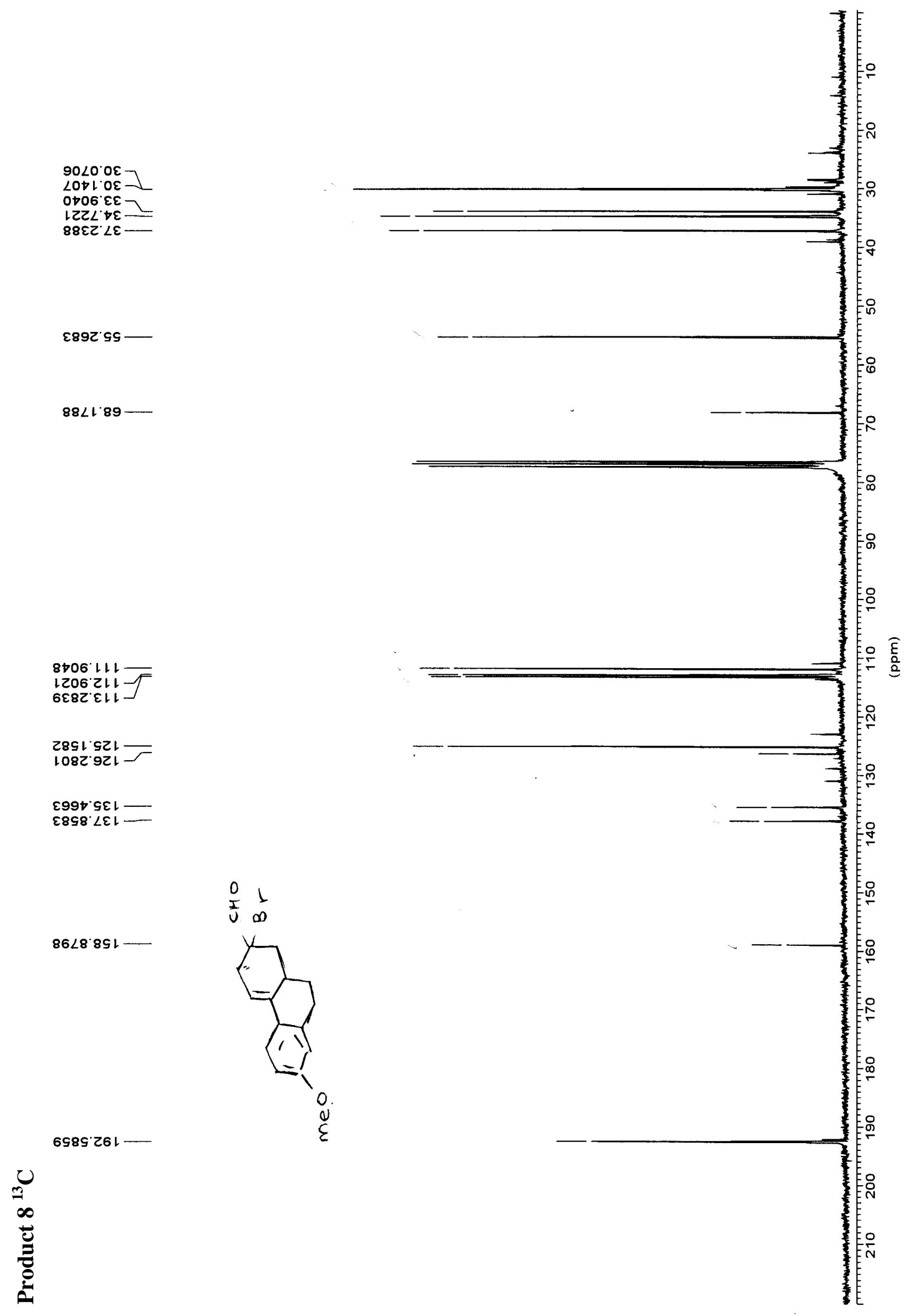




\section{Product 8 HPLC spectrum}

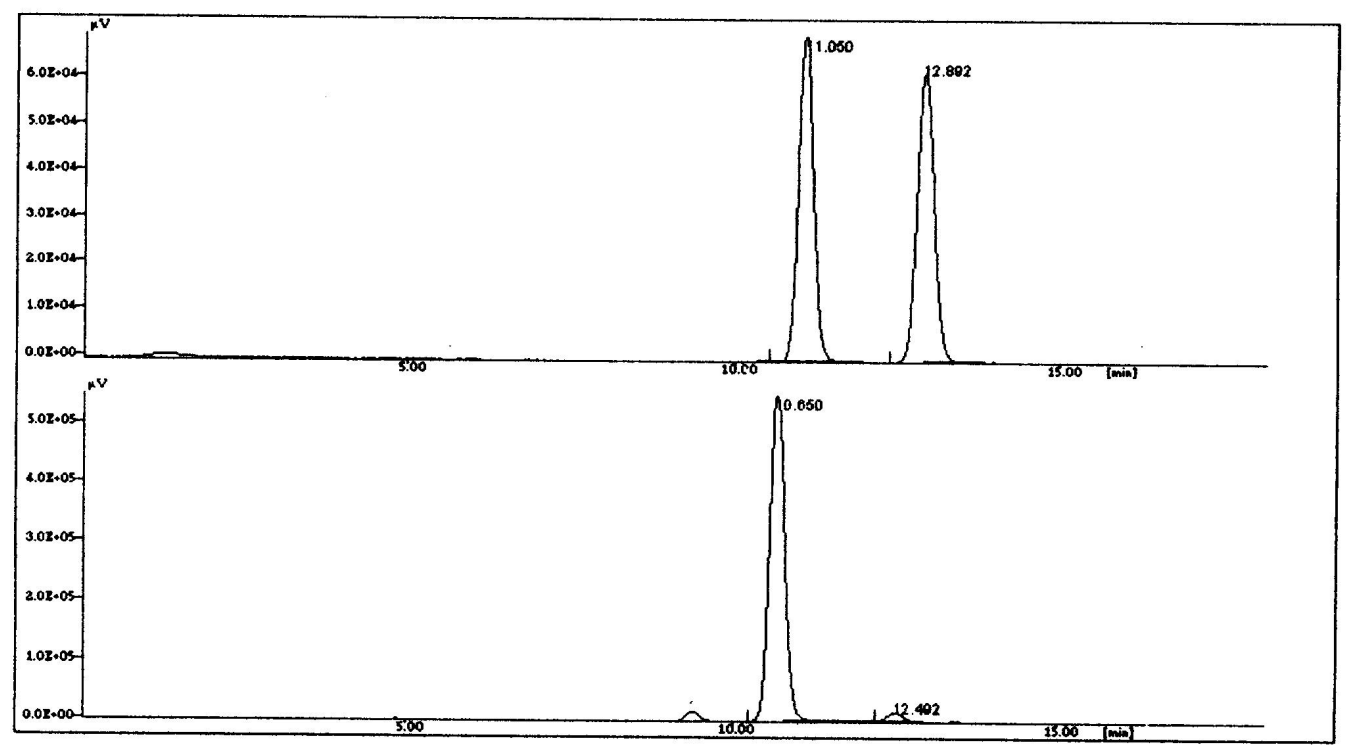

Info :

da, ad-H, all,chiral

$\begin{array}{cccccc}\# & \text { Name } & \text { RT } & \text { Area }[\mu \text { V.Sec }] & \text { \%Area Corr. Area } & \text { Are } \\ 1 & & 10.650 & 8062546.524 & 97.27 & 8062546.52 \\ 2 & & 12.492 & 1.724 & 0.00 & 225929.25\end{array}$

Total Area of Peak $=8288475.774[\mu$ V.Sec $]$<smiles>C=C[C@]12CC=CC(CCc3cc(OC)ccc3C1)C2</smiles> 